\title{
Evolution of Asymmetries in Vortex Breakdown
}

\author{
Andrew W. Cary*, David L. Darmofal ${ }^{\dagger} \quad$ Kenneth G. Powell ${ }^{\ddagger}$ \\ University of Michigan, Ann Arbor, Michigan 48105
}

\begin{abstract}
The influence of weak asymmetric disturbances on an axisymmetric swirling flow is examined through numerical simulation. To track the evolution of asymmetries, the incompressible Navier-Stokes equations are Fourier-decomposed in the azimuthal direction and integrated forward in time. Beginning with an axisymmetric solution, an asymmetric disturbance is added at the inlet of a converging-diverging nozzle and allowed to propagate. For axisymmetric flows which do not exhibit vortex breakdown, the disturbance is merely convected and dissipated. The presence of axisymmetric breakdown, however, amplifies the disturbance and large changes occur in the flow structures. Depending on the axisymmetric swirl ratio, non-axisymmetric solutions range from no apparent breakdown, to the spiral mode, and, at larger swirl ratios, to an oscillation between the spiral mode and the bubble mode. For swirl ratios corresponding to a weak axisymmetric breakdown, asymmetry creates a "relieving effect" which eliminates the recirculation zone, resulting in a smooth solution with only weak asymmetries.
\end{abstract}

${ }^{*}$ University of Michigan, presently at The Boeing Company, Professional Member AIAA.

${ }^{\dagger}$ Professor, Department of Aerospace Engineering, Texas A \& M University, Professional Member AIAA.

${ }^{\ddagger}$ Professor, Department of Aerospace Engineering, University of Michigan, Senior Member AIAA.

Copyright (C)1998 by Andrew Cary. Published by the American Institute of Aeronautics and Astronautics, Inc. with permission.

\section{Introduction}

The rapid transition of a vortex from a coherent spinning fluid to a more diffuse state that rapidly decays represents a fluid dynamic problem that poses interesting academic questions and has bearing on many problems of practical interest, from maneuvering at high angles of attack to decreasing landing distances. While first observed experimentally over 40 years ago ${ }^{1}$, most of the attempts to analyze this phenomenon have necessarily assumed axisymmetry. Until recent advances in computational power, consideration of the full three-dimensional unsteady problem has been impractical. The sensitivity of the flow to experimental probes has also hindered investigation of this phenomenon. Most of the experimental studies have concentrated on measuring the flow far upstream or downstream of breakdown and inferring the flow structure from flow visualization. While these techniques have identified several modes of breakdown, the most common are the nearly axisymmetric bubble breakdown and the spiral mode. In the bubble mode of breakdown, the vortex core suddenly swells, develops a roughly axisymmetric recirculation zone, and quickly decays into turbulence. Most of the experiments have indicated that while the outer envelope of this mode appears axisymmetric, the internal flow has significant asymmetrical velocities. The spiral mode is characterized by a rapid turning of the vortex core away from its axis, followed by several helical twists before becoming turbulent.

Despite the limitations that axisymmetry puts on the flow, both simulations and theoretical developments have contributed a number of useful 
AIAA 98-2904

concepts describing vortex breakdown. While many of the classical results are discussed thoroughly in review papers by Hall ${ }^{2}$ and Leibovich ${ }^{3}$, there have been several significant recent extensions. Darmofal ${ }^{4}$ described vortex breakdown as a space-time focusing of disturbance waves which accumulate at the axial location where the flow first becomes critical and allows standing waves. Rusak ${ }^{5}$ has obtained a map of solutions to the incompressible, inviscid, axisymmetric equations of motion and demonstrated that, for a specific range of swirl ratios, multiple solutions exist which represent both columnar and breakdown flows. Once the columnar flow becomes critical, it becomes unstable to axisymmetric perturbations and transitions to the alternate solution branch containing vortex breakdown.

This paper examines the limitations of axisymmetric theory for breakdown flows by simulating an axisymmetric flow field and subjecting it to a weak asymmetric disturbance. The effect of this disturbance is examined and characterized as a function of swirl ratio for flows in a convergingdiverging nozzle.

\section{Approach}

The incompressible Navier-Stokes equations are written in cylindrical coordinates and Fourierdecomposed in the azimuthal direction to obtain a sequence of coupled partial differential equations:

$$
\begin{gathered}
\frac{\partial \mathbf{u}_{0}}{\partial t}+\mathbf{U} \cdot \nabla_{0} \mathbf{u}_{0}+\mathbf{u}_{0} \cdot \nabla_{0} \mathbf{U}+\mathbf{u}_{0} \cdot \nabla_{0} \mathbf{u}_{0}+ \\
\sum_{m=1}^{N}\left(\mathbf{u}_{m} \cdot \nabla_{-m} \overline{\mathbf{u}}_{m}+\overline{\mathbf{u}}_{m} \cdot \nabla_{m} \mathbf{u}_{m}\right)= \\
-\nabla_{0} p_{0}+\frac{1}{R e} \nabla_{0}^{2} \mathbf{u}_{0}
\end{gathered}
$$

and

$$
\begin{gathered}
\frac{\partial \mathbf{u}_{m}}{\partial t}+\mathbf{U} \cdot \nabla_{m} \mathbf{u}_{m}+\mathbf{u}_{m} \cdot \nabla_{0} \mathbf{U}+\mathbf{u}_{0} \cdot \nabla_{m} \mathbf{u}_{m}+ \\
+\mathbf{u}_{m} \cdot \nabla_{0} \mathbf{u}_{0}+\sum_{j=1}^{m-1} \mathbf{u}_{j} \cdot \nabla_{m-j} \mathbf{u}_{m-j} \\
+\sum_{j=1}^{N_{g}-m}\left(\mathbf{u}_{m+j} \cdot \nabla_{-j} \overline{\mathbf{u}}_{j}+\overline{\mathbf{u}}_{j} \cdot \nabla_{m+j} \mathbf{u}_{m+j}\right) \\
=-\nabla_{m} p_{m}+\frac{1}{R e} \nabla_{m}^{2} \mathbf{u}_{m},
\end{gathered}
$$

where

$$
\nabla_{m} f(r, z)=e^{\imath m \theta} \nabla\left(f(r, z) e^{-\imath m \theta}\right)
$$

and an overbar () denotes complex conjugation.

Additionally, the Fourier-decomposed perturbation velocity must conserve mass,

$$
\nabla_{m} \cdot \mathbf{u}_{m}=0 \quad \forall m \geq 0 .
$$

The Fourier-decomposition of the velocity is specified by

$$
\begin{aligned}
& \mathbf{u}=\mathbf{U}(r, z)+\mathbf{u}_{0}(r, z, t)+ \\
& \sum_{m=1}^{N_{\theta}}\left(\mathbf{u}_{m}(r, z, t) e^{-\imath m \theta}+\overline{\mathbf{u}}_{m}(r, z, t) e^{\imath m \theta}\right)
\end{aligned}
$$

where $\mathbf{U}(r, z)$ is a steady axisymmetric base solution. Thus $\mathbf{u}_{m}=u_{m} \mathbf{e}_{r}+v_{m} \mathbf{e}_{\theta}+w_{m} \mathbf{e}_{z}$ is a complex function of $r$ and $z$ representing the $m$ th azimuthal mode of the perturbation velocity. This approach both allows for more efficient calculation of the lower azimuthal modes that are more dominant in experiments and parallels many theoretical investigations of the flow, supporting and providing guidance for additional investigations.

These equations are discretized through the use of Chebyshev collocation in the radial direction and finite-differencing in the axial direction. The resulting system of equations is solved using an operator split approach that successively integrates the flow in time by performing a non-linear advection step, a pressure correction, and a viscous correction 6,7 . Following previous numerical investigations, the flow is simu- 
lated in a converging-diverging nozzle that isolates the breakdown from the inlet boundary conditions. A slip wall boundary condition is applied on the nozzle to prevent the formation of a boundary layer that would separate and interact with the breakdown. This choice for a boundary condition prevents this interaction that would complicate the basic physics being examined and roughly simulates an axisymmetric streamtube of a flow in a pressure gradient. The inflow boundary specifies the velocity upstream of the throat as a free stream flow with an azimuthal velocity given by

$$
V(r, z=0)=\frac{\omega}{r}\left(1-e^{-r^{2}}\right),
$$

where $\omega$ is the swirl ratio.

\section{Axisymmetric results}

Beginning with a non-swirling flow through the nozzle depicted in Figure 2, the swirl ratio is slightly increased and the flow is allowed to converge. Using this approach, the swirl ratio is incremented to higher and higher values until breakdown occurs. The Reynolds number based on core radius for the solutions presented in this paper is 500 , high enough that viscous effects do not dominate, yet low enough to exclude broadband turbulence. This approach develops a family of axisymmetric solutions which can be perturbed at the inlet by a weak asymmetric disturbance. One of the advantages of solving the Fourier-decomposed system is that the flow can be constrained to remain axisymmetric until an asymmetry is introduced. This allows the same procedure to be used for both axisymmetric and fully three-dimensional solutions, enabling a direct comparison of results and detailed information regarding the evolution of the different azimuthal modes.

In this particular geometry, the axisymmetric solutions can be characterized by the minimum axial velocity on the axis, as is depicted in Figure 1. At a swirl ratio of about 1.48 , this quantity becomes negative, a recirculation zone exists in the fluid, and axisymmetric vortex breakdown is predicted. For sufficiently high swirls,

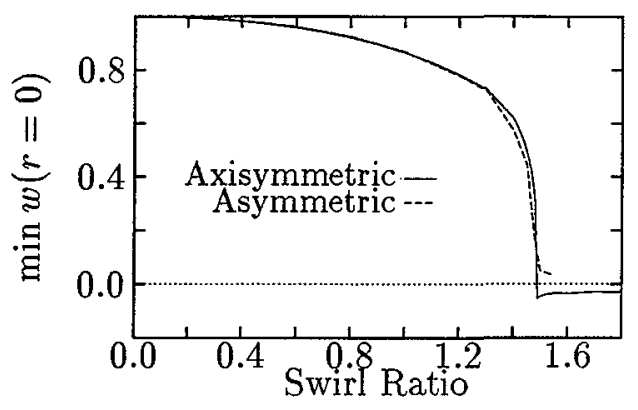

Figure 1: Minimum axial velocity at $r=0$ for axisymmetric and asymmetric solutions parameterized by swirl ratio.

the minimum axial velocity begins to increase on the axis, even though a recirculation region still exists in the flow. This is due to the forward migration of the aft end of the breakdown bubble until a deformed recirculation toroid exists. This solution evolution is depicted in Figure 2.

\section{Asymmetric Results}

Beginning with an axisymmetric base flow obtained using the above approach, a weak disturbance of the form

$$
w_{1}(r, z=0)=A \frac{r^{2}}{R^{2}} e^{-12 r^{2} / R^{2}}
$$

is introduced to the $m=1$ mode of the axial velocity, where $R$ is the radius of the inlet and $A$ is a measure of the amplitude. This amplitude is linearly ramped in time to 1 percent of the inlet axial velocity and allowed to convect and interact with the flow. This perturbation, depicted in Fig. 3, is chosen to satisfy the boundary condition at the axis and to decay rapidly outside the vortex core. As a consequence of the physical requirement for single-valued functions at $r=0$, only the $m=1$ mode can deflect fluid off the axis. Since this mode can also stimulate all other azimuthal modes, it provides an effective means for introducing asymmetry into the flow. To ensure that the significant effects are resolved in these calculations, between 2 and 5 asymmetric modes are simulated. Comparison 


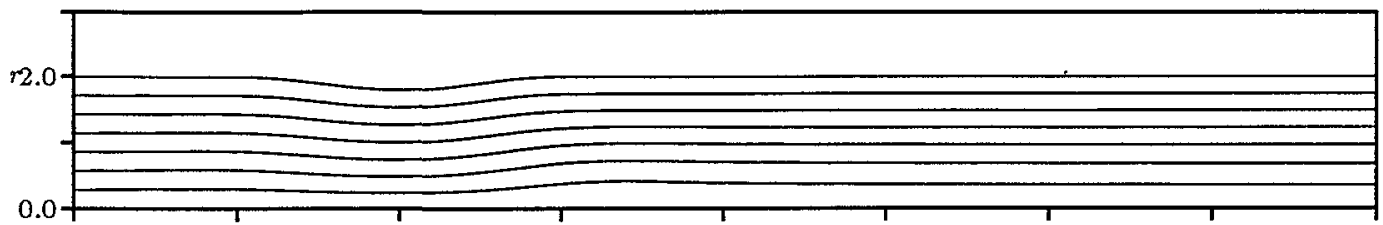

a. Swirl ratio $=1.47$

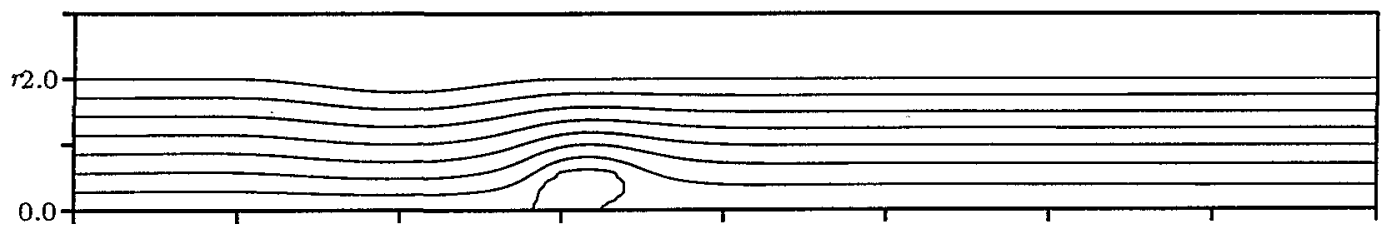

b. Swirl ratio $=1.49$

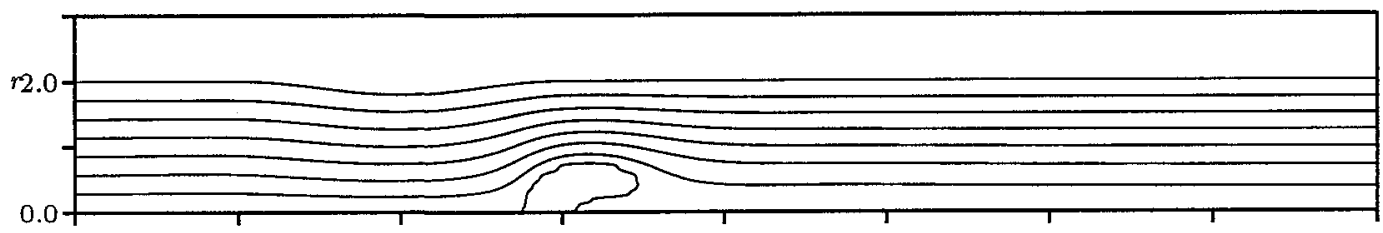

c. Swirl ratio $=1.51$

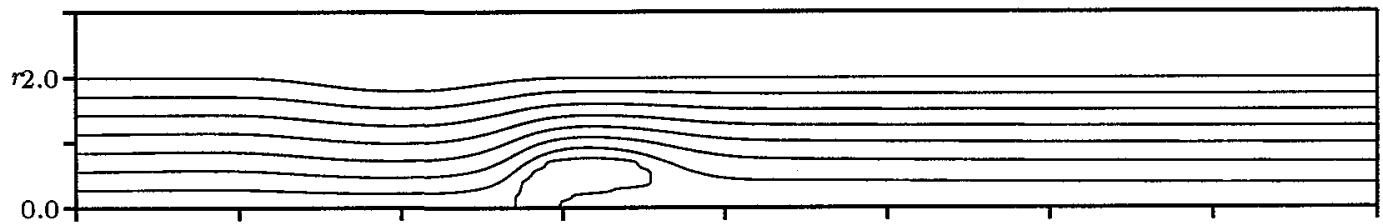

d. Swirl ratio $=1.53$

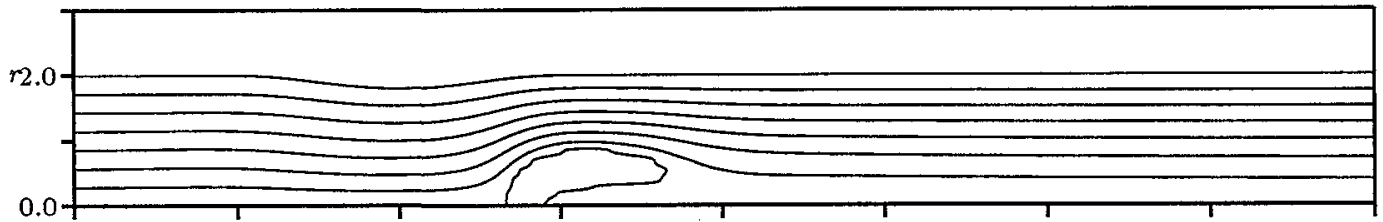

e. Swirl ratio $=1.55$

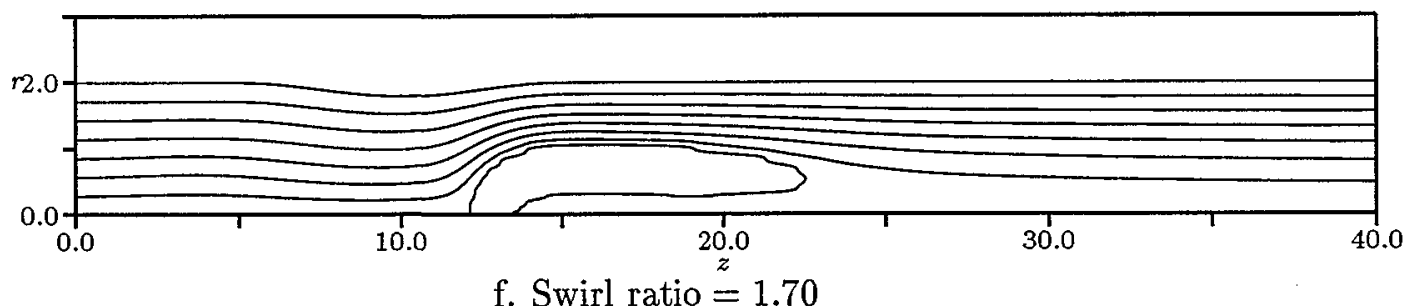

Figure 2: Development of axisymmetric breakdown with swirl ratio. 


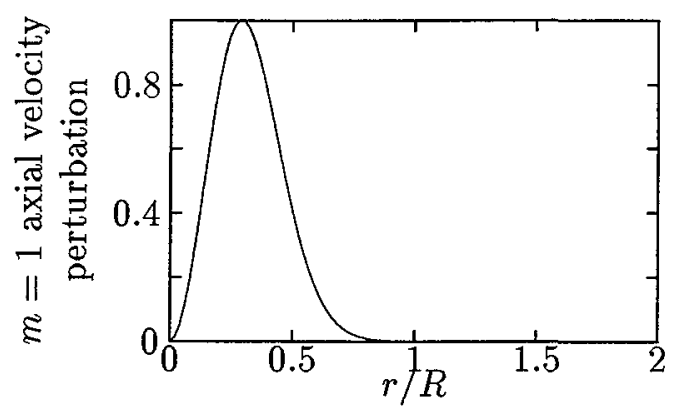

Figure 3: Radial distribution of axial velocity perturbation mode.

of the different resolutions indicate that the dominant features of the flow appear in the lowest several modes. ${ }^{7}$

The degree that the perturbation affects the flow can be evaluated by considering the integrated mode magnitude,

$$
Q_{m}=\int_{\mathcal{V}} \frac{1}{2} \mathbf{u}_{m} \cdot \overline{\mathbf{u}}_{m} \mathrm{~d} r \mathrm{~d} z,
$$

where $\mathcal{V}$ is a radial slice through the nozzle.

Evolution equations ${ }^{7}$ reveal that the integrated mode magnitude can only be changed by viscous dissipation, non-linear interactions among perturbation modes, focusing of perturbations by the base strain rate, and boundary effects. For each mode, the viscous dissipation always leads to a decrease. The non-linear interactions of each mode can be identically matched to terms with opposite sign in other modes. Thus, while energy is transferred between modes, it does not lead to an overall increase in the strength of the asymmetries. For the axisymmetric mode, this term can be related to the work required to maintain the asymmetry and tends to initially decrease the axisymmetric velocities. This "energy transfer" has been suggested by Leibovich and Kribus $^{8}$ as an additional dissipation that can stabilize their axisymmetric soliton solution. The remaining term,

$$
-\Re \int_{\mathcal{V}} \overline{\mathbf{u}}_{m} \cdot \nabla_{0} \mathbf{U}_{0} \cdot \mathbf{u}_{m} \mathrm{~d} \mathcal{V}
$$

is the only term that can increase the total magnitude represented by the sum of all $Q_{m}$. This

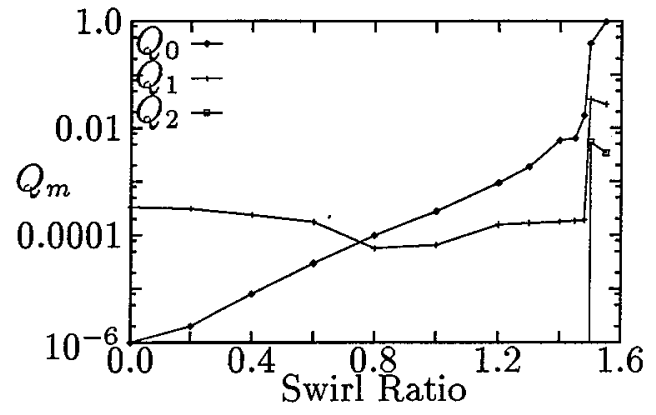

Figure 4: Variation of $Q_{m}$ with axisymmetric swirl ratio for $1 \% m=1$ axial velocity perturbation.

focusing of asymmetric modes by the axisymmetric strain rate of the base flow leads to perturbation growth in regions where the base flow tends to be compressing in the direction of the perturbation velocity.

For the flows with axisymmetric swirl ratios less than that leading to breakdown, the perturbation does not show significant amplification and decays with axial distance. This behavior is reflected in Figure 4 which depicts the variation in $Q_{m}$ with swirl ratio for the converged asymmetric solution. The slight decrease in magnitude for swirl ratios less than the critical swirl ratio leading to breakdown is due to increased viscous dissipation. For a non-swirling flow, the disturbance propagates directly down the pipe. The addition of swirl both causes the disturbance to spiral as it travels downstream and shears the perturbation, increasing the dissipation rate. For these solutions, the weak asymmetry gradually decays and does not have any significant effect on the axisymmetric flow. The base flows at these lower swirl ratios have much lower strain rates than flows with axisymmetric breakdown.

As demonstrated by the small values of $Q_{m}$ in Fig. 4 and by the agreement between the axisymmetric and asymmetric solution curves in Fig. 1, significant asymmetries do not develop until a swirl ratio of 1.49. At this swirl ratio, the axisymmetric flow undergoes vortex breakdown and a stagnation point develops on the axis. The region near the leading edge of the recirculation 
zone has both large strain rates and decelerated flow that both tend to amplify asymmetric disturbances. In these cases, the perturbation is amplified by several orders of magnitude (see Fig. 4) and leads to large changes in the flow field. These changes are immediately evident in Figure 1 which indicates that the asymmetric solution does not develop a stagnation point on the axis even after the axisymmetric solution is predicting breakdown. While asymmetric solutions have been calculated in this geometry for swirl ratios up to 1.8 , these higher swirl cases have not converged to a steady solution, but continue to have large, nearly periodic, oscillations for long times.

Figures 5 and 6 depict the evolution of the flow with swirl ratio 1.55 after the perturbation is introduced at the inlet. Figure 5 suggests that there are large oscillations in the nature of the solution beginning at about $t=40$ nondimensional times $\left(t U_{\infty} / \delta\right)$. At this point, the asymmetries have convected to the breakdown bubble and are beginning to be amplified. This is also reflected in the temporal evolution of the minimum axial velocity on the axis. This quantity shows large fluctuations both positive and negative as the stagnation point moves off the axis, vanishes, and reappears. These changes are also clearly evident in the vortex core images of Figure 6 . Initially, the bubble is seen to grow, both in velocity magnitude and size, with the significant asymmetric velocity confined to the recirculation zone. The asymmetries continue to grow in time and push the stagnation point off the axis, deflecting the incoming flow and leading to the characteristic corkscrew of a spiral breakdown. As the stagnation point continues to wander away from the axis, the flow is deflected less strongly and the recirculation zone weakens. This leads to a decrease in the asymmetries and the stagnation point reappears near the axis, again increasing the asymmetric nature of the flow. As the stagnation point gets close enough to the axis, the helix angle of the spiral is increased and the nearly axisymmetric bubble mode reappears.

This cycle continues with diminishing amp-
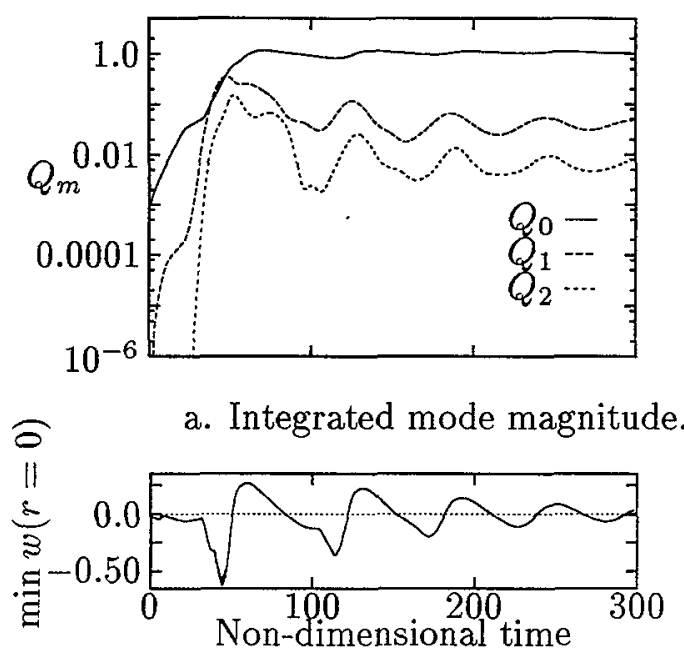

b. Minimum axial velocity on axis.

Figure 5: Development of integrated mode magnitudes and minimum axial velocity at $r=0$ as a function of time for swirl ratio $=1.55$.

litude for a considerable time. Eventually, the solution converges to an approximately steady flow with no stagnation point. While asymmetry remains, it does not amplify significantly because of the much smaller strain rate in the axisymmetric component of the solution. This threedimensional solution closely resembles a continuation of the columnar solution branch which exists for purely axisymmetric flows just before breakdown. Thus, while Wang and Rusak ${ }^{9}$ have demonstrated that axisymmetric flows with swirl ratios greater than the critical swirl ratio (i.e. subcritical flows) are unstable to axisymmetric disturbances, this result indicates that these flows may be stabilized by the introduction of asymmetric components. This result has also been obtained by solutions in a different geometry with several different perturbation magnitudes ${ }^{7}$. The additional degrees of freedom created by removing the axisymmetric constraint provides a relieving effect that allows the non-breakdown solution to be extended to at least slightly higher swirl ratios.

Simulations at a higher swirl ratio of 1.70 show a behavior similar to these results, but after 400 non-dimensional time units, a periodic solu- 
AIAA 98-2904

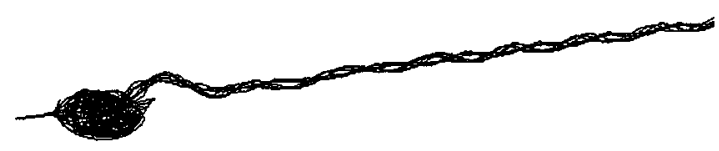

a. $t=40$

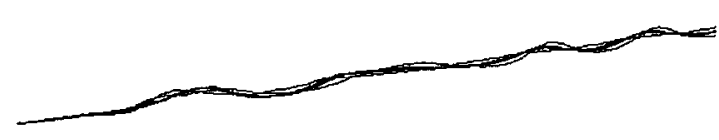

b. $t=60$

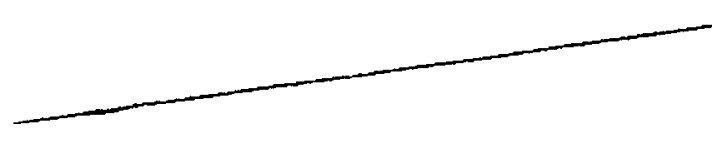

c. $t=80$

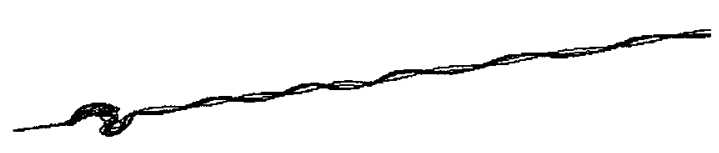

d. $t=110$

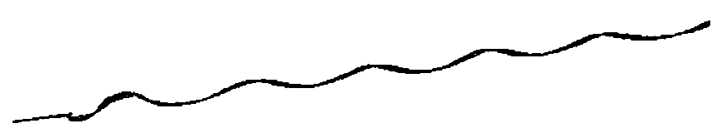

c. $t=120$

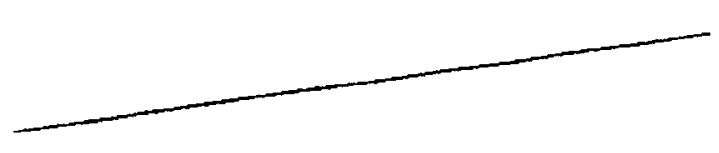

d. $t=140$

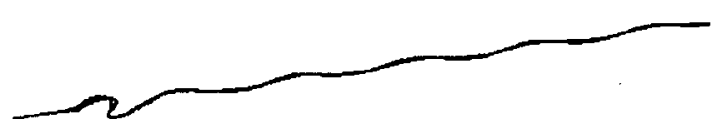

d. $t=170$

Figure 6: Instantaneous streamlines of flow at swirl ratio of 1.55 after asymmetric perturbation. tion exists with no indication of damping. The asymmetries begin to magnify when the initial disturbance convects to the large axisymmetric strain rates in the neighborhood of the breakdown bubble. Initially, this increase causes the recirculation region to grow and propagate slightly upstream before the periodic behavior is established. Seven cycles with a period of about 37 non-dimensional time units were simulated and no indication of damping was observed.

\section{Description}

One of the classical theories for axisymmetric breakdown is based on the criticality of the flow ${ }^{10}$. For sufficiently low swirl ratios, the flow is supercritical and axisymmetric perturbation waves propagate only downstream. At the critical swirl ratio, standing waves are permitted on the base solution and breakdown results from the accumulation of these standing waves. Using axisymmetric numerical solutions, Darmofal ${ }^{4}$ calculated the minimum disturbance wave speeds and found that the downstream convective speed of perturbations gradually decreased as a recirculation bubble was approached. This focusing of disturbances in an axisymmetric flow increases the local strain and provides a mechanism for the growth of asymmetries. The simulations discussed above indicate that the asymmetries can grow strong enough to significantly affect the axisymmetric flow by moving the stagnation point off the axis. The rapid growth of asymmetries once the critical swirl ratio is reached suggests that the space-time focusing of axisymmetric waves is required for amplification of asymmetric disturbances.

As originally described by Brown and Lopez ${ }^{11}$ and further developed by Darmofal ${ }^{4}$, the possibility of a feedback process leading to breakdown exists for axisymmetric flows. By definition, negative azimuthal vorticity $\left(\eta_{0}\right)$ is kinematically associated with deceleration of the axial velocity at $r=0$. As the axial component of vorticity is tilted by a small divergence of the flow, the azimuthal component of vorticity is decreased. This serves to further decelerate the flow on the 
axis, increasing both divergence and the tilting of the vorticity vector. This process has the capability of rapidly stagnating the flow on the axis and creating breakdown. Neglecting viscosity to leading order in a asymmetric flow specifies that the axisymmetric azimuthal velocity will change according to

$$
\begin{aligned}
\frac{\mathrm{D} \eta_{0}}{\mathrm{D} t} & =\xi_{0} \frac{\partial v_{0}}{\partial r}+\zeta_{0} \frac{\partial v_{0}}{\partial z}-\frac{v_{0} \xi_{0}}{r}+\frac{u_{0} \eta_{0}}{r} \\
& +2 \sum_{m=1}^{\infty} \Re\left(\bar{\xi}_{m} \frac{\partial v_{m}}{\partial r}+\bar{\zeta}_{m} \frac{\partial v_{m}}{\partial z}\right. \\
& \left.-\frac{v_{m} \bar{\xi}_{m}}{r}+\bar{\eta}_{m} \frac{u_{m}+\imath m v_{m}}{r}\right)
\end{aligned}
$$

where the vorticity is $\boldsymbol{\omega}=\xi \mathbf{e}_{r}+\eta \mathbf{e}_{\theta}+\zeta \mathbf{e}_{z}$. The first three terms of both the axisymmetric and the non-axisymmetric contributions are due to tilting of the vorticity vector into the azimuthal direction, while the final term in each group stems from vortex stretching. After the flow is perturbed by an asymmetric disturbance, the growth of the asymmetric azimuthal vorticity $\left(\eta_{1}\right)$ is driven by asymmetric stretching of the axisymmetric azimuthal vorticity as well as stretching by the divergence. While $\eta_{0}$ decelerates the flow on the axis, $\eta_{1}$ tends to deflect the flow off the axis. In the present case, this asymmetry feeds back into the evolution of $\eta_{0}$ via the stretching term and has a stabilizing effect as the flow is directed off-axis. While the near-stagnant flow remains near the axis, the incoming flow is deflected and a spiral breakdown occurs. This deflection decreases the size of the recirculation zone and reduces the axisymmetric strain rate, damping the growth of the asymmetric modes. As a consequence of these effects, stationary waves are no longer supported and the recirculation region disappears. However, once the stagnation point is pushed far enough away from the axis, the axisymmetric vorticity is stretched less and the tilting mechanism again dominates, leading to flow stagnation and the reestablishment of a recirculation zone.

\section{Conclusion}

Numerical simulations of the evolution of weak asymmetric perturbations on a base axisymmetric flow are performed using spectral techniques to solve the incompressible Navier-Stokes equations. To parallel theoretical analyses, these equations have been Fourier-decomposed in the azimuthal direction. These simulations reveal that the assumption of axisymmetry is reasonable until breakdown is predicted. When this happens, a large strain rate field develops that tends to amplify asymmetric perturbations, leading to significant changes in the character of the solution. These changes can result in the elimination of the recirculation zone or the development of the spiral breakdown mode. The existence of nonbreakdown flows at subcritical swirl ratios suggests the possibility that the axisymmetric columnar solution branch may be extended by allowing weak asymmetric disturbances, even though axisymmetric solutions at these higher swirl ratios are unstable. The present simulations trace the development of flow asymmetry on a base axisymmetric flow and capture both the spiral and bubble modes of vortex breakdown. Using these data, the evolution of breakdown can be described by the motion of the stagnation point about the axis. For weakly subcritical flows, the initial recirculation zone collapses with the addition of asymmetry, while larger swirl ratios lead to more complicated flow evolutions which include oscillations between both spiral and bubble breakdown modes. The kinematics of this oscillation can be described by considering the feedback of negative azimuthal vorticity. While negative axisymmetric azimuthal vorticity tends to stagnate the flow, the presence of asymmetric ( $m=1$ mode) azimuthal vorticity tends to push the stagnation point off the axis. These two trends compete against each other and, depending on the swirl ratio, can lead to a weaklyasymmetric nearly-columnar flow, the spiral mode of breakdown, the bubble breakdown mode, or quasi-periodic combinations of these solutions. 
AIAA 98-2904

\section{References}

1 D. H. Peckham and S. A. Atkinson. Preliminary results of low speed wind tunnel tests on a gothic wing of aspect ratio 1.0. CP 508, Aeronautics Research Council, 1957. 16 pp.

2 M. G. Hall. Vortex breakdown. Annual Review of Fluid Mechanics, 4:195-217, 1972.

3 Sidney Leibovich. Vortex stability and breakdown: Survey and extension. AIAA Journal, 22:1192-1206, 1983.

4 David L. Darmofal. A Study of the Mechanisms of Axisymmetric Vortex Breakdown. $\mathrm{PhD}$ thesis, Massachusetts Institute of Technology, November 1993.

5 S. Wang and Z. Rusak. A theory of the axisymmetric vortex breakdown in a pipe. Aeronautical Engineering Report 107, Rensselaer Polytechnic Institute, Troy, New York, February 1995. To appear in Journal of Fluid Mechanics.

6 Andrew W. Cary, David L. Darmofal, and Kenneth G. Powell. Onset of the spiral mode of vortex breakdown. AIAA 97-0439. 35th Aerospace Sciences Meeting. January 1997.

7 Andrew W. Cary. The Onset of NonAxisymmetric Vortex Breakdown. $\mathrm{PhD}$ thesis, University of Michigan, Ann Arbor, MI, August 1997.

8 Sidney Leibovich and A. Kribus. Largeamplitude wavetrains and solitary waves in vortices. Journal of Fluid Mechanics, 216:459-504, 1990.

9 S. Wang and Z. Rusak. On the stability of an axisymmetric rotating flow in a pipe. Physics of Fluids, 8:1007-1016, 1996.

$10 \mathrm{H}$. B. Squire. Analysis of the "vortex breakdown" phenomenon: part I. Report 102, Aero. Dept., Imperial College, London, 1960. Also in Miszellaneen der Angewandten Mechanik, pp. 306-312, 1962. Berlin: AkademieVerlag.
11 G. L. Brown and J. M. Lopez. Axisymmetric vortex breakdown. part 2. physical mechanisms. Journal of Fluid Mechanics, 221:553$576,1990$. 